\title{
Histoplasmose intestinal em pacientes transplantados renais: relato de casos e revisão da literatura
}

\section{Intestinal histoplasmosis in renal transplanted patients: case reports and literature review}

Francisco Daniel Bezerra Amorim ${ }^{1}$. Isabela Orieta de Oliveira Macedo ${ }^{1}$. Evelyne Santana Girão ${ }^{1}$. Sonia Leite da Silva ${ }^{1,2}$. Paula Frassinetti Castelo Branco Camurça Fernandes ${ }^{1}$. Claudia Maria Costa de Oliveira ${ }^{1,3}$.

1 Hospital Universitário Walter Cantídio (HUWC), Universidade Federal do Ceará (UFC), Fortaleza, Ceará, Brasil. 2 Universidade de Fortaleza (UNIFOR), Fortaleza, Ceará, Brasil. 3 Centro Universitário Christus (Unichristus), Fortaleza, Ceará, Brasil.

\section{RESUMO}

A histoplasmose é uma micose sistêmica, que afeta principalmente pacientes imunocomprometidos, com apresentação clínica inespecífica. A forma de apresentação é focal ou disseminada, esta última muitas vezes fatal. Os autores relatam dois casos de histoplasmose intestinal em pacientes transplantados renais, com baixo índice de suspeição clínica, que foram diagnosticados através de biópsia das lesões e que apresentaram uma evolução favorável, devido ao tratamento precoce com anfotericina B lipossomal e manutenção com itraconazol. Diante de casos com manifestações clínicas variadas e atípicas, esta doença deve ser incluída na investigação diagnóstica de imunossuprimidos.

Palavras-chave: Histoplasmose. Transplante renal. Biopsia.

\section{ABSTRACT}

Histoplasmosis is a systemic mycosis, affecting mainly immunocompromised patients with nonspecific clinical presentation. The presentation form is focal or widespread, the latter often fatal. The authors report two cases of intestinal histoplasmosis in renal transplant patients, with a low rate of clinical suspicion, who were diagnosed by lesion biopsy and showed a favorable outcome due to early treatment with liposomal amphoterecin $b$ and maintenance with itraconazole. In the face of cases with varied and atypical clinical manifestations, this disease should be included in the diagnostic investigation of immunosuppressed individuals.

Keywords: Histoplasmosis. Kidney transplantation. Biopsy.

Autor correspondente: Francisco Daniel Bezerra Amorim, Rua do Manguesais, 60, Dionísio Torres, Fortaleza, Ceará. CEP: 60135-640. Telefone: +55 88 99967-4607. E-mail: daniel_bamorim@hotmail.com

Conflito de interesses: Não há qualquer conflito de interesses por parte de qualquer um dos autores.

Recebido em: 11 Set 2019; Revisado em: 23 Jan 2020; Aceito em: 01 Out 2020. 


\section{INTRODUÇÃO}

A histoplasmose é uma micose sistêmica que afeta principalmente pacientes imunocomprometidos, com formas clínicas focal ou disseminada, esta última muitas vezes fatal. Essa doença era predominante em pacientes com neoplasias e transplantados renais até a década de 80 , no Brasil. Com o surgimento da síndrome da imunodeficiência adquirida, houve um aumento no número de casos de histoplasmose, principalmente na forma disseminada. ${ }^{1,2}$

É causada por um fungo dimórfico, o Histoplasma capsulatum, que apresenta duas variedades: o Histoplasma capsulatum var. capsulatum e o Histoplasma capsulatum var. duboisii. Estes patógenos são encontrados no solo contendo fezes de aves e morcegos, permanecendo neste ambiente por longo período. $^{2}$

Trata-se de uma micose endêmica na América Latina, nos Estados Unidos, parte da Ásia e África. Têm ocorrido epidemias de histoplasmose aguda em áreas endêmicas e não endêmicas após a exposição a ambientes contaminados com o fungo, como por exemplo cavernas onde moram morcegos, galinheiros e telhados de casas abandonadas. No Brasil, foram observados surtos nos Estados de São Paulo, Rio de Janeiro, Espírito Santo, Mato Grosso e Minas Gerais. ${ }^{2}$

A histoplasmose pode apresentar um quadro com sintomas respiratórios (50\% dos casos) ou de forma disseminada $(75 \%) .^{3}$ A histoplasmose disseminada é rara e os casos são mais descritos em pacientes com SIDA. Receptores de transplante renal podem ter uma manifestação atípica, devido à imunossupressão. ${ }^{4}$

Nos receptores de transplante de órgão sólido, a infecção pode ocorrer de forma primária, secundária à exposição prévia ou reativação de infecção latente. A transmissão através do aloenxerto é rara. ${ }^{5}$

Os autores relatam dois casos clínicos de histoplasmose intestinal em receptores de transplante renal, que raramente vem para atenção clinica por falta de sintomas específicos. Tratandose de uma doença endêmica e sendo a imunossupressão um fator de risco, o diagnóstico precoce torna-se essencial, uma vez que pode impactar na morbimortalidade do paciente. Neste artigo, queremos trazer o foco para a importância da inclusão desta micose no diagnóstico diferencial em transplantados renais com manifestações atípicas, e mostrar o seu desfecho clínico. O estudo foi submetido ao CEP do Hospital Universitário Walter Cantídio e aprovado com o número de parecer 2.174.880.

\section{RELATO DE CASOS}

\section{Caso 1}

Homem, 67 anos, transplante renal com doador falecido em 2015, doença de base indeterminada, fez terapia imunossupressora de indução com timoglobulina e manutenção com tacrolimo, micofenolato sódico e prednisona.
Comorbidade prévia ao transplante: doença pulmonar intersticial por provável comprometimento ocupacional (mineração).

Em agosto de 2018, apresentou perda de peso (cinco quilos em 2 meses), associada a estímulo gastrocólico exacerbado, sem febre, diarreia, sangramento nas fezes ou outros sintomas. Realizou uma colonoscopia, em outubro de 2018, evidenciando duas úlceras rasas, uma no ceco próxima à válvula íleocecal $(3 \mathrm{~cm})$ e outra no colón descendente $(7 \mathrm{~mm})$. As lesões biopsiadas mostraram reação granulomatosa colônica com aumento do número de eosinófilos intersticiais, presença de estruturas ovalas isoladas ou em grupamentos, que coraram pelo Grocott (Figura 1), e ausência de BAAR. Os achados histológicos em análise conjunta com a histoquímica, foram sugestivos de histoplasmose.

Figura 1. Estruturas ovaladas sugestivas de fungo, Grocott, 400x.

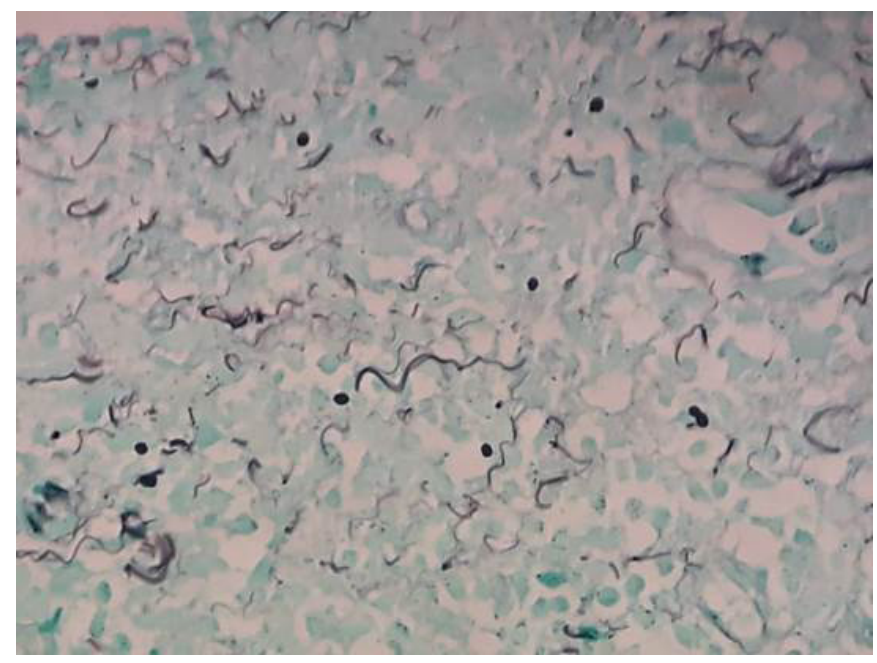

Uma tomografia de tórax de alta resolução (TCAR) foi realizada para investigar outros sítios de infecção fúngica, mostrando opacidades reticulares subpleurais, de forma mais acentuada nos lobos inferiores, associadas a bronquioloectasias de tração, sugerindo intersticiopatia fibrosante. A pesquisa para BAAR e cultura do lavado broncoalveolar foram negativas. A biópsia transbrônquica revelou uma bronquiolite obliterante com pneumonia em organização.

Foi iniciado tratamento com anfotericina B lipossomal por 14 dias, com elevação da creatinina (creatinina basal de 1,6 mg/ dl, chegando a $4,2 \mathrm{mg} / \mathrm{dl}$ ) por nefrotoxicidade medicamentosa. Devido ao aumento da creatinina, foi realizada biópsia do enxerto renal, sem sinais de rejeição. A imunossupressão foi convertida de tacrolimo para everolimo e a dose de prednisona foi aumentada de 5 para $40 \mathrm{mg} / \mathrm{dia}$, com posterior redução gradual. A terapia antifúngica de manutenção foi o itraconazol oral, por doze meses. Evoluiu com melhora clínica e da função renal após terapia antifúngica. 
Uma colonoscopia de controle foi realizada em janeiro de 2019, evidenciando duas úlceras rasas, com base recoberta por tênue camada de fibrina, uma adjacente à válvula ileocecal $(4 \mathrm{~mm})$ e outra no colón ascendente $(2 \mathrm{~mm})$. O histopatológico não mais evidenciou estruturas compatíveis com histoplasma.

\section{Caso 2}

Homem, 59 anos, transplante renal com doador falecido em junho de 2008, doença de base nefroesclerose hipertensiva, indução com basiliximabe e manutenção com tacrolimo, micofenolato mofetil e prednisona. $\mathrm{Na}$ evolução póstransplante, apresentava disfunção do enxerto (clearance de creatinina $<60 \mathrm{ml} / \mathrm{min} / \mathrm{m}^{2}$ ) e uma biópsia renal revelou presença de fibrose intersticial e atrofia tubular (IFTA) em $20 \%$ do fragmento, compatíveis com nefropatia crônica do enxerto.

Em novembro de 2018, apresentou quadro de edema, calor e rubor de membro inferior direito, associado à febre, e uma ultrassonografia de partes moles da perna direita demonstrou aumento da ecogenicidade e perda parcial do padrão fibrilar dos músculos da face ântero-lateral, achados compatíveis com miosite. Foi internado e iniciado, empiricamente, vancomicina, porém, a resposta clínica não foi satisfatória e o esquema de antibiótico foi escalonado para meropenem e linezolida. Evoluiu com piora da função renal (creatinina basal de 2,3 mg/dl), com início de hemodiálise.

No internamento, apresentou quadro de melena e endoscopia digestiva alta evidenciou edema de mucosa gástrica e pré-pilórica e úlceras duodenais. A biópsia das úlceras revelou processo inflamatório intenso, mucosa duodenal com macrófagos infestados por estruturas esporiformes intracitoplasmáticas, que impregnaram em negro ao método de Grocott (Figura 2), confirmando histoplasmose duodenal.

Figura 2. Histoplasma realçados pela prata. Coloração Grocott, 400x.

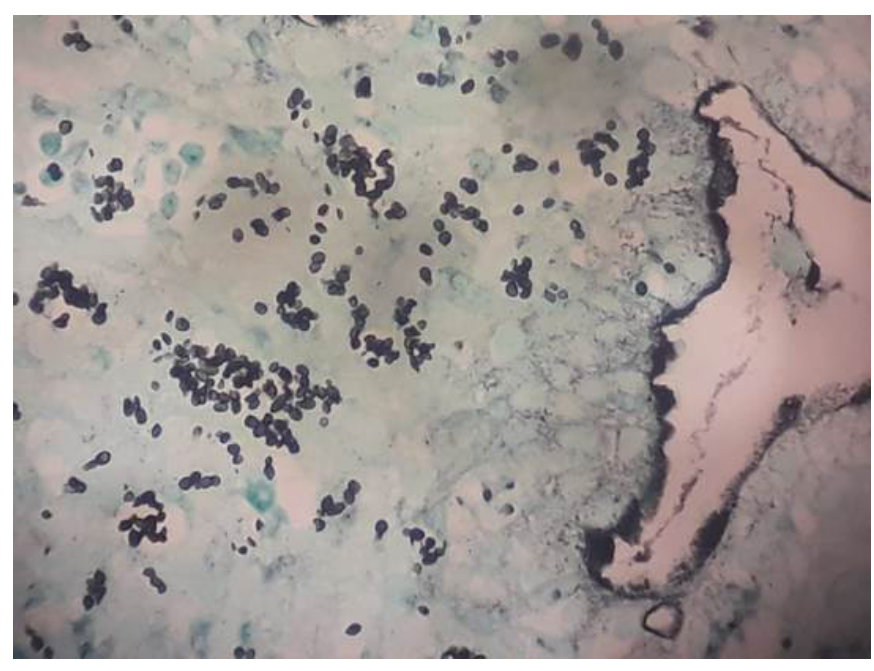

Uma TCAR mostrou micronódulos com distribuição randômica, além de nódulos acinares multifocais nas porções médias e superiores. As hipóteses de tuberculose ou histoplasmose em suas formas miliares foram consideradas. A broncoscopia foi normal e a pesquisa de BAAR e Genexpert negativas no lavado broncoalveolar. Uma biópsia transbrônquica não foi realizada. Vale ressaltar que o paciente não apresentava evidências de imunossupressão excessiva ou de infecção oportunista prévia.

Foi tratado com anfotericina B lipossomal por 10 dias, havendo melhora da lesão do membro inferior direito, sendo considerada a infecção cutânea como também de etiologia fúngica. Foi mantida terapia com itraconazol $200 \mathrm{mg} /$ dia, por doze meses. Não houve recuperação da função renal, sendo encaminhado para clínica de hemodiálise.

\section{DISCUSSÃO}

A histoplasmose disseminada se desenvolve em pacientes com imunodeficiência, sendo caracterizada pela sua apresentação extrapulmonar. $\mathrm{O}$ acometimento intestinal está presente em 70 a $90 \%$ dos casos disseminados e cerca de $70 \%$ das autópsias revelam algum envolvimento gastrointestinal. ${ }^{6}$ Nos pacientes imunocompetentes, essa manisfestação raramente é relatada. ${ }^{7}$

A clínica da doença intestinal é descrita como sangramento, ulcerações, obstrução e perfuração intestinal em pacientes imunocomprometidos, podendo ser também assintomática. As manifestações gastrointestinais mais relatadas são dor e distensão abdominal em $75 \%$ dos casos. ${ }^{8}$ As manifestações inespecíficas como febre, perda de peso, sudorese noturna, calafrios e diarreia, são descritas em menos de $10 \%$ dos casos de histoplasmose disseminada. ${ }^{9}$

Os autores relatam dois casos de histoplasmose gastrointestinal, um deles com localização no trato gastrointestinal superior e com melena e outro com doença em região colônica, com manifestação gastrocólica exarcebada. Nestes casos, o quadro clínico foi de sintomas gastrointestinais mais discretos. A histoplasmose gastrointestinal pode acometer qualquer porção do trato digestório, entretanto cerca de $90 \%$ dos casos envolve a região ileocecal ou cólon, ${ }^{6}$ como relatado no primeiro caso. Talvez isto se deva à abundância de tecido linfoide associado ao intestino (GALT) nestas áreas, o que funciona como sítio de entrada para macrófagos repletos de esporos de Histoplasma capsulatum. ${ }^{10}$ Em uma grande série de casos de histoplasmose gastrointestinal em pacientes com HIV, a doença duodenal foi observada em menos de $4 \%$ dos casos, sendo um sítio raro de envolvimento do trato gastrointestinal. ${ }^{11}$ Em transplante renal, os poucos casos publicados são acometem o colón, ${ }^{12}$ sendo importante ressaltar que esse parece ser um dos primeiros casos de histoplasmose duodenal relatado em transplante renal.

A suspeita clínica de histoplasmose foi importante para o diagnóstico e tratamento precoces. Sua forma de apresentação disseminada apresenta uma taxa de mortalidade de 31\% em pacientes imunocomprometidos e $17 \%$ em pacientes imunocompetentes. ${ }^{13}$ É importante incluir histoplasmose 
na impressão diagnóstica em imunossuprimidos de áreas endêmicas, devido sua apresentação muitas vezes inespecífica e que confunde com outras patologias, além de sua elevada mortalidade.

Vale ressaltar que no segundo caso, a apresentação clínica inicial foi de miosite em membro inferior. A suspeita clínica inicial foi de miosite bacteriana, não tendo havido resposta aos diferentes esquemas antibióticos. Não foi realizado biópsia muscular e cultura de fragmento muscular para identificação do fungo por falta de suspeição clínica, mas após o diagnóstico de histoplasmose duodenal, o paciente evoluiu com franca melhora da miosite com a introdução de anfotericina. A miosite por Histoplasma capsulatum tem sido raramente descrita, mesmo em pacientes com HIV, receptores de transplante e pacientes com doenças reumatológicas e sua evolução tem sido descrita como fatal nos casos relatados, com envolvimento miofascial necrotizante e disseminação fúngica. ${ }^{14-16}$

Embora as manifestações clínicas da histoplasmose já tenham sido bem descritas, o diagnóstico não pode ser alcançado somente clinicamente, podendo ocorrer sobreposição com outras doenças. Para o diagnóstico, é importante a identificação do histoplasma em fluidos corporais ou biópsia de tecido, cultura de espécimes clínicos e biológicos (a qual confere diagnóstico definitivo), ensaios sorológicos, detecção de antígenos, testes cutâneos ou métodos de biologia molecular. ${ }^{17}$ Nos casos do presente relato, o diagnóstico foi por biópsia de tecido. Vale ressaltar que na endoscopia digestiva alta, as lesões macroscópicas no trato gastrointestinal são semelhantes a outras patologias, como neoplasias e doenças inflamatórias intestinais. ${ }^{6} \mathrm{O}$ sistema respiratório nos dois casos relatados também foi examinado, mas não houve envolvimento pulmonar no primeiro caso ou manifestação clínica pulmonar no segundo caso. Na análise histopatológica da medula óssea, pulmão, linfonodos, intestino, mucosas e pele, na evidência de granulomas epitelioides de aspectos sarcoide, os microorganismos podem ser visualizados no interior de células fagocíticas através da coloração de Gomori-Grocott. ${ }^{18}$

\section{REFERÊNCIAS}

1. Fernandes AR, Viana LA, Mansur JB, Françoso MM, Santos DW, Silva HT, et al. Histoplasmose disseminada em uma paciente transplantada renal. J Bras Nefrol. 2018;40(1):95-7.

2. Ferreira MS, Borges AS. Histoplasmose. Rev Soc Bras Med Trop. 2009;42(2):192-8.

3. Fishman JA. Infection in solid-organ transplant recipients. N Engl J Med. 2007;357(25):2601-14.

4. Simon DM, Levin S. Infectious complications of solid organ transplantations. Infect Dis Clin North Am. 2001;15(2):521-49.

5. Assi M, Martin S, Wheat LJ, Hage C, Freifeld A, Avery R, et al. Histoplasmosis after solid organ transplant. Clin Infect Dis. 2013;57(11):1542-9.

6. Spinner MA, Paulin HN, Wester CW. Duodenal histoplasmosis
A anfotericina B é a medicação de escolha para o tratamento, destruindo a membrana celular do microorganismo. A dose total recomendada é de $35 \mathrm{mg} / \mathrm{kg}$ (sendo de 2,0 a 2,5g a dose total). Trata-se de uma droga tóxica, sendo os principais efeitos adversos a nefrotoxicidade, náuseas, vômitos e cefaleia. A remissão completa do quadro clínico é de cerca de $75 \% .^{2}$ Apesar da formulação lipossomal ser descrita como menos nefrotóxica, os pacientes deste relato apresentaram nefrotoxicidade pelo uso da medicação e a disfunção renal prévia à infecção pode ter contribuído para a piora de função renal durante o seu uso. ${ }^{19} \mathrm{O}$ paciente do primeiro caso teve recuperação da função renal ao seu valor basal e o paciente do segundo caso necessitou de diálise antes do início da anfotericina $\mathrm{B}$, mas a sua utilização pode ter contribuído para a persistência da disfunção renal pós-tratamento.

A manutenção a longo prazo da terapia é importante para remissão clínica, sendo que uma opção é a anfotericina B na dose de $1 \mathrm{mg} / \mathrm{kg}$, duas vezes por semana, endovenosa, mas o itraconazol oral é eficaz nesta situação e deve ser mantido por um ano, sendo a terapia preferida quando disponível. ${ }^{2}$

\section{CONCLUSÃO}

Foram relatados dois casos de histoplasmose intestinal em transplante renal, com baixa suspeição clínica, que foram diagnosticados através de exames invasivos, respondendo satisfatoriamente ao tratamento. Um dos casos foi de histoplasmose duodenal que não tem sido previamente descrita em transplante renal, associada à miosite fúngica, manifestação também pouco usual neste grupo de pacientes.

A histoplasmose gastrointestinal deve ser considerada no diagnóstico diferencial de sangramento gastrointestinal em receptores de transplante renal que vivem em áreas endêmicas, bem como reflexo gastrocólico exacerbado. O diagnóstico precoce de histoplasmose é de extrema importância para uma evolução clínica favorável, principalmente em pacientes imunocomprometidos.

presenting with upper gastrointestinal bleeding in an AIDS patient. Case Rep Gastrointest Med. 2012;2012:515872.

7. Zhu LL, Wang J, Wang ZJ, Wang YP, Yang JL. Intestinal histoplasmosis in immunocompetent adults. World J Gastroenterol. 2016;22(15):4027-33.

8. Yang B, Lu L, Li D, Liu L, Huang L, Chen L, et al. Colonic involvement in disseminated histoplasmosis of an immunocompetent adult: case report and literature review. BMC Infect Dis. 2013;13:143.

9. Kane S, Brasitus T. Histoplasmosis capsulatum as a cause of lower gastrointestinal bleeding in common variable immunodeficiency. Dig Dis Sci. 2000;45(11):2133-5.

10. Haws CC, Long RF, Caplan GE. Histoplasma capsulatum as a cause of ileocolitis. Am J Roentgenol. 1977;128(4):692-4. 
11. Assi M, McKinsey DS, Driks MR, O'Connor MC, Bonacini M, Graham B, et al. Gastrointestinal histoplasmosis in the acquired immunodeficiency syndrome: report of 18 cases and literature review. Diagn Microbiol Infect Dis. 2006;55(3):195-201.

12. Syed TA, Salem G, Kastens DJ. Lower gastrointestinal bleeding secondary to intestinal histoplasmosis in a renal transplant patient. ACG Case Rep J. 2017;4:e93.

13. Sathapatayavongs B, Batteiger BE, Wheat J, Slama TG, Wass JL. Clinical and laboratory features of disseminated histoplasmosis during two large urban outbreaks. Medicine (Baltimore). 1983;62(5):263-70.

14. Nimitvilai S, Thammaprasert W, Vinyuvat S. Histoplasmosis myositis: a case report and literature review. Southeast Asian J Trop Med Public Health. 2015;46(4):738-42.
15. Voloshin DK, Lacomis D, McMahon D. Disseminated histoplasmosis presenting as myositis and fasciitis in a patient with dermatomyositis. Muscle Nerve. 1995;18(5):531-5.

16. Wagner JD, Prevel CD, Elluru R. Histoplasma capsulatum necrotizing myofascitis of the upper extremity. Ann Plast Surg. 1996;36(3):330-3.

17. Guimarães AJ, Nosanchuk JD, Zancope-Oliveira RM. Diagnosis of histoplasmosis. Braz J Microbiol. 2006;37(1):1-13.

18. Wheat LJ. Diagnosis and management of histoplasmosis. European Journal of Clin Microbiol Infect Dis. 1989;8:480-90.

19. Botero Aguirre J, Restrepo Hamid A. Amphotericin B deoxycholate versus liposomal amphotericin B: effects on kidney function. Cochrane Database Syst Rev. 2015;23(11):CD010481.

\section{Como citar:}

Amorim FD, Macedo IO, Girão ES, Silva SL, Fernandes PF, Oliveira CM. Histoplasmose intestinal em pacientes transplantados renais: relato de casos e revisão da literatura. Rev Med UFC. 2020 out-dez;60(4):49-53. 